\title{
ASSISTENZA INFERMIERISTICA PER PAZIENTE CON PIEDE DIABETICO
}

\section{ARTICOLO ORIGINALE}

CUNHA, Amanda Priscilla da ${ }^{1}$

CUNHA, Amanda Priscilla da. Assistenza infermieristica per paziente con piede diabetico. Revista Científica Multidisciplinar Núcleo do Conhecimento. Anno. 06, Ed. 12, Vol. 12, pp. 111-126. Dicembre 2021. ISSN: 2448-0959, Link di accesso: https://www.nucleodoconhecimento.com.br/salute/piede-diabetico, DOI: 10.32749/nucleodoconhecimento.com.br/salute/piede-diabetico

\section{RIEPILOGO}

Introduzione: II piede diabetico chiamato anche da uno stato fisiopatologico sfaccettato è caratterizzato da lesioni che derivano dai piedi della persona con diabete senza un trattamento adeguato e cure specifiche. Si stima che l'incidenza dell'ulcera del piede diabetico raggiunga il $6,3 \%$ dei pazienti diabetici e la sua prevalenza si avvicini al $10 \%$ e le persone con basso status socioeconomico sono le maggiori vittime. Alla luce di queste evidenze, il lavoro si basa sulla seguente domanda problematica: quale cura sviluppano gli infermieri nei pazienti con piedi diabetici? Obiettivo generale: Evidenziare la cura del piede diabetico correlata alla pratica degli infermieri. Metodologia: Una revisione integrativa è stata condotta nel Database bibliografico specializzato in infermieristica (BDENF), nella letteratura latinoamericana e caraibica nelle scienze della salute (LILACS) e nella Scientific Electronic Library Online (SCIELO) e che ha portato a sette articoli. Risultati: Gli studi hanno mostrato come la cura degli infermieri con educazione alla salute del piede diabetico, formazione continua e permanente, tecniche di valutazione al piede diabetico che comportano esami fisici e auto-cura dei pazienti con piedi diabetici. È rafforzato il fatto che questa cura dovrebbe essere eseguita in via prioritaria dagli

\footnotetext{
${ }^{1}$ Master in gestione Ospedaliero e Sistemi Sanitari, Infermiera Laurea in Infermieristica. ORCIDE: 000-00015456-1848.
}

RC: 105165

Disponibile in: https://www.nucleodoconhecimento.com.br/salute/piede-diabetico 
infermieri, poiché gli infermieri hanno un'assistenza specifica e qualificata al paziente nelle loro principali attribuzioni. Conclusione: Si conclude, quindi, che lo studio ha identificato diverse forme di assistenza fornite dagli infermieri e che tutte queste forme sono importanti per la continuità del trattamento e la prevenzione delle complicanze, tuttavia, spetta allinfermiere essere un professionista che valorizza la qualità delle proprie cure sulla base di concetti teorici che portano a una formazione frequente.

Parole chiave: Assistenza al paziente, diabete mellito, piede diabetico.

\section{INTRODUZIONE}

Il diabete mellito (DM) è una malattia sviluppata nel sistema endocrino e/o un disturbo metabolico cronico, che rappresenta un grave problema di salute in tutto il mondo e ha come caratteristica principale l'iperglicemia, raggiungendo un livello di glucosio nel sangue superiore a $126 \mathrm{mg} / \mathrm{dL}$ a digiuno $0>200 \mathrm{mg} / \mathrm{dL}$ nella fase post prandiale, e che può essere stato associato all'insulino-resistenza e che può essere stato associato all'insulino-resistenza, inadeguata secrezione di insulina o eccessiva secrezione di glucagone (ASCHNER et al., 2016).

Pertanto, l'iperglicemia causa l'incapacità totale o parziale della produzione di insulina, secondo la Società brasiliana del diabete (SBD), l'American Diabetes Association (ADA) e l'Organizzazione Mondiale della Sanità (OMS) DM è diviso in quattro gruppi: diabete mellito di tipo 1 , diabete mellito di tipo 2 , diabete mellito gestazionale e altri tipi specifici di diabete (OMS, 1999; SBD, 2017; ADA 2017).

SBD descrive i fattori di rischio per DM, che sono citati come segue: diagnosi di prediabete - diminuzione della tolleranza al glucosio o alterato glucosio a digiuno; ipertensione; ipercolesterolemia o cambiamenti nel tasso di trigliceridi nel sangue; sovrappeso e obesità, soprattutto se il grasso è concentrato intorno alla vita; storia familiare; bambino che pesa più di quattro chili o diabete gestazionale; sindrome dell'ovaio policistico; diagnosi di alcuni disturbi psichiatrici, come la schizofrenia, la depressione, il disturbo bipolare e l'uso di farmaci psicotropi; altri (SBD, 2018). 
Trattandosi di una malattia che colpisce circa il 3\% della popolazione mondiale e con una prevalenza approssimativa del $6,2 \%$ della popolazione in Brasile, la cui prevalenza è aumentata a causa dell'invecchiamento della popolazione (MUZY et al., 2021), il DM ha causato un onere sulle casse pubbliche, oltre a consentire anche costi elevati per svolgere la cura delle sue complicanze quali: piede diabetico, malattie cardiovascolari, amputazioni e altro. Vale la pena ricordare che il trattamento farmacologico ha fatto progressi nel controllo del DM, tuttavia l'educazione sanitaria come forma di prevenzione deve essere trasmessa alla popolazione generale individualmente $\mathrm{o}$ in gruppo, sottolineando il problema della malattia e i suoi danni alla salute ( SILVA, 2018).

Per quanto riguarda le complicanze del DM, il piede diabetico è anche chiamato per il suo stato fisiopatologico sfaccettato, caratterizzato da lesioni che derivano dai piedi della persona con diabete da neuropatia nel $90 \%$ dei casi, in cui la malattia vascolare periferica e le deformità sono predominanti (CUBAS et al., 2017).

De Sousa Mendonça; Moraes; Moura (2017) describe che le lesioni esistenti nel piede diabetico provengono dalla combinazione di due o più fattori di rischio che possono essere correlati a traumi intrinseci come estrinseci, associati a neuropatia periferica che causa perdita di sensibilità, facilitando il rischio di tagli; Malattia vascolare periferica derivante da riduzione del flusso sanguigno negli arti inferiori e alterazione biomeccanica che si verifica quando vi è restrizione dei movimenti delle articolazioni del piede e della caviglia.

Si stima che l'incidenza dell'ulcera del piede diabetico raggiunga il 6,3\% dei pazienti diabetici e la sua prevalenza si avvicini al $10 \%$ e le persone con basso status socioeconomico sono le maggiori vittime. L'amputazione del piede diabetico è un altro problema e che in tutto il mondo ogni tre minuti una persona ha il suo arto amputato a causa di DM (ARRUDA et al., 2021).

La buona gestione dei fattori coinvolti nell'eziologia della lesione esistente nel piede diabetico diventa efficace quando eseguita dall'infermiere, quindi l'infermiere deve essere qualificato e qualificato per eseguire la consultazione infermieristica al fine di 
non vedere cambiamenti dermatologici, muscoloscheletrici, vascolari e neurologici nel piede diabetico (FÉLIX et al., 2021).

Pertanto, la cura dei piedi diabetici diventa molto importante all'interno della sanità pubblica e l'infermiere, come professionista che ha nella sua premessa la cura del paziente, dovrebbe essere attento e qualificato per quanto riguarda le nuove forme di trattamento per ridurre le amputazioni. Di fronte a questo scenario di tassi allarmanti, è essenziale osservare come gli infermieri si trovano nelle pratiche di cura dei piede diabetico.

Data questa evidenza che coinvolge numeri allarmanti in relazione al piede diabetico, il lavoro si basa sulla seguente domanda problematica: quale cura sviluppano gli infermieri nei pazienti con piede diabetico?

Lo studio in questione mira a dimostrare la cura del piede diabetico correlata alla pratica degli infermieri.

\section{METODOLOGIA}

Per questo studio, è stata condotta una revisione integrativa della letteratura. Secondo Mendes; Silveira e Galvão (2019), il metodo di revisione integrativa viene sviluppato seguendo passaggi ben definiti; sono: identificazione del tema e selezione della questione settentrionale; stabilire criteri di inclusione ed esclusione; identificazione di studi preselezionati e selezionati; categorizzazione di studi selezionati; analisi e interpretazione dei risultati e presentazione della sintesi della revisione.

Il primo passo della revisione integrativa è la questione settentrionale, che porta al tema e agli obiettivi elencati. Pertanto, la domanda che sorge in questo articolo è: quale cura sviluppano gli infermieri nei pazienti con piede diabetico?

Per stabilire i criteri di inclusione sono stati utilizzati: articoli pubblicati e disponibili per la lettura, poiché l'argomento riflette l'infermiere nelle loro attribuzioni, è stato scelto il Database di BDENF, LILACS e SCIELO, la delimitazione temporale è stata 
negli anni dal 2016 al 2020, cioè negli ultimi cinque anni, e la lingua portoghese è stata scelta per questa inclusione.

Per i criteri di esclusione sono stati rimossi: articoli che hanno doppiezza, articoli incompleti, annais, testi di opinione, editoriali e lettere al lettore, articoli di recensione, tesi, tesi, testi completi, ma che non riguardano il tema.

Il periodo di raccolta dei dati corrispondeva al mese di settembre 2021. I descrittori nazionali individuati sono stati: "assistenza infermieristica", "piede diabetico", "infermiere", "diabete mellito". L'operatore booleano and è stato utilizzato per associare i descrittori nella strategia di ricerca sulla piattaforma di database.

Per la selezione di studi selezionati e preselezionati, è stata effettuata una lettura approfondita dei titoli e degli abstract degli articoli trovati, e quindi una lettura più completa in tutto l'articolo, cercando nei suoi risultati l'identificazione per raggiungere l'obiettivo proposto in questo articolo.

Sono state effettuate due ricerche, la prima ha utilizzato la seguente strategia di ricerca: assistenza infermieristica and piede diabetico and diabete mellito, con questa strategia sono stati trovati 186 articoli. Dopo aver utilizzato i filtri: testo completo e disponibile, database, lingua portoghese e delimitazione temporale degli ultimi cinque anni, 24 articoli sono rimasti ammissibili. La seconda ricerca è stata eseguita con la seguente strategia: infermiera and piede diabetico trovando 95 articoli. Dopo aver utilizzato i filtri, 11 articoli sono rimasti idonei.

Gli studi sono stati classificati da un quadro sinottico e l'analisi e la presentazione dei risultati è stata condotta dopo la categorizzazione che ha identificato e definito $i$ nuclei di significato degli studi, e che dall'analisi tematica di Minayo (2012), c'è stata una discussione tra gli autori trovati.

Per la sintesi delle conoscenze, è stata effettuata un'analisi dei limiti, della rappresentatività dello studio per la comunità in generale (rilevanza) e degli studi futuri identificati nel corso di questo articolo. Tutte le produzioni utilizzate in questo 
studio sono rigorosamente referenziate secondo gli standard dell'Associazione brasiliana degli standard tecnici (ABNT).

\section{RISULTATI E DISCUSSIONI}

Gli articoli rimasti per l'ammissibilità alle due strategie ammontano a 35 articoli. Inizialmente, gli articoli di revisione, i lavori di conclusione del corso, le lettere al lettore, i rapporti sull'esperienza, i casi di studio, gli articoli duplicati sono stati rimossi e che nei loro titoli, abstract e letture per intero non hanno portato l'infermiere e la sua assistenza come tema all'argomento, lasciando 07 articoli per questa recensione.

Per una migliore illustrazione dei risultati trovati, la seguente tabella includerà la caratterizzazione degli studi selezionati. I dati si riferiscono a: titolo dell'articolo, anno di pubblicazione, database, rivista, posizione, obiettivo e risultati.

Tavolo uno. Articoli trovati nel database BDENF, LILACS e SCIELO sull'assistenza infermieristica per pazienti con piedi diabetici.

\begin{tabular}{|c|c|c|c|c|c|}
\hline $\begin{array}{l}\text { Titolo/ Anno } \\
\text { di } \\
\text { pubblicazione }\end{array}$ & $\begin{array}{l}\text { banca } \\
\text { dati }\end{array}$ & $\begin{array}{l}\text { periodic } \\
0\end{array}$ & Traguardo & Locale & Risultati \\
\hline $\begin{array}{l}\text { Conheciment } \\
0 \quad \text { do } \\
\text { enfermeiro } \\
\text { acerca dos } \\
\text { cuidados com } \\
\text { o pé diabético } \\
2019\end{array}$ & BDENF & $\begin{array}{l}\text { Rev. } \\
\text { enferm. } \\
\text { UFPE } \\
\text { on line }\end{array}$ & $\begin{array}{l}\text { Comprendere } \\
\text { le conoscenze } \\
\text { dell'infermiere } \\
\text { sulla cura del } \\
\text { piede diabetico } \\
\text { nelle cure } \\
\text { primarie }\end{array}$ & $\begin{array}{l}\text { Strategia } \\
\text { per la } \\
\text { salute della } \\
\text { famiglia/FS } \\
\text { E }\end{array}$ & $\begin{array}{l}\text { Eseguire } \\
\text { periodicamente } \\
\text { l'esame fisico } \\
\text { dei piedi nei } \\
\text { diabetici; } \\
\text { Uso di } \\
\text { strumenti per la } \\
\text { valutazione del } \\
\text { piede diabetico } \\
\text { Cura di sé ai } \\
\text { piedi }\end{array}$ \\
\hline
\end{tabular}




\begin{tabular}{|c|c|c|c|c|c|}
\hline $\begin{array}{l}\text { mortalidade } \\
\text { em pacientes } \\
\text { atendidos em } \\
\text { um programa } \\
\text { de prevenção }\end{array}$ & $\begin{array}{l}\text { LILACS } \\
/ \\
\text { BDENF }\end{array}$ & $\begin{array}{l}\text { Rev. } \\
\text { gaúch. } \\
\text { enferm }\end{array}$ & $\begin{array}{l}\text { Identificare nei } \\
\text { pazienti con } \\
\text { diabete di tipo } \\
2 \quad \text { quali } \\
\text { cambiamenti } \\
\text { nei piedi } \\
\text { sarebbero } \\
\text { associati alle } \\
\text { caratteristiche } \\
\text { demografiche, } \\
\text { cliniche, } \\
\text { biochimiche e } \\
\text { di trattamento } \\
\text { e quali di essi } \\
\text { aumenterebber } \\
\text { o il rischio di } \\
\text { mortalità }\end{array}$ & $\begin{array}{l}\text { Programma } \\
\text { educativo } \\
\text { per la } \\
\text { prevenzion } \\
\text { e e il } \\
\text { trattamento } \\
\text { del piede } \\
\text { diabetico in } \\
\text { un } \\
\text { ospedale } \\
\text { generale e } \\
\text { universitari } \\
\text { o di alta } \\
\text { complessità }\end{array}$ & $\begin{array}{l}\text { Educazione } \\
\text { sanitaria } \\
\text { sull'igiene del } \\
\text { piede, la scelta } \\
\text { di calzature } \\
\text { adeguate, la } \\
\text { migliore } \\
\text { condotta in } \\
\text { situazioni di } \\
\text { emergenza. } \\
\text { Cure } \\
\text { sistemiche } \\
\text { come non } \\
\text { fumare, } \\
\text { mantenere un } \\
\text { buon controllo } \\
\text { glicemico, } \\
\text { controllare } \\
\text { tassi di lipidi nel } \\
\text { sangue e l'uso } \\
\text { corretto dei } \\
\text { farmaci }\end{array}$ \\
\hline $\begin{array}{l}\text { Condutas dos } \\
\text { enfermeiros } \\
\text { da atenção } \\
\text { primária no } \\
\text { cuidado a } \\
\text { pessoas com } \\
\text { pé diabético } \\
2017\end{array}$ & BDENF & $\begin{array}{l}\text { Rev. } \\
\text { enferm. } \\
\text { UFPE } \\
\text { on line }\end{array}$ & $\begin{array}{l}\text { Conoscere le } \\
\text { azioni degli } \\
\text { infermieri di } \\
\text { assistenza } \\
\text { primaria nella } \\
\text { cura delle } \\
\text { persone con } \\
\text { diabete mellito } \\
\text { (DM) correlato }\end{array}$ & $\begin{array}{l}\text { Strategia } \\
\text { per la } \\
\text { salute della } \\
\text { famiglia }\end{array}$ & $\begin{array}{l}\text { Frequenza e } \\
\text { programmazion } \\
\mathrm{e} \quad \text { delle } \\
\text { consultazioni, } \\
\text { dinamiche di } \\
\text { servizio, criteri } \\
\text { per l'esame del } \\
\text { piede, cura di } \\
\text { sé }\end{array}$ \\
\hline
\end{tabular}




\begin{tabular}{|c|c|c|c|c|c|}
\hline & & & $\begin{array}{l}\text { al piede } \\
\text { diabetico }\end{array}$ & & \\
\hline $\begin{array}{l}\text { Ações do } \\
\text { enfermeiro na } \\
\text { prevenção do } \\
\text { pé diabético: } \\
\text { o olhar da } \\
\text { pessoa com } \\
\text { diabetes } \\
\text { mellitus } \\
2017\end{array}$ & $\begin{array}{l}\text { LILACS } \\
/ \\
\text { BDENF }\end{array}$ & $\begin{array}{l}\text { Rev. } \\
\text { Pesqui. } \\
\text { (Univ. } \\
\text { Fed. } \\
\text { Estado } \\
\text { Rio J., } \\
\text { Online) }\end{array}$ & $\begin{array}{l}\text { Indagare le } \\
\text { azioni svolte } \\
\text { dagli infermieri } \\
\text { nella } \\
\text { prevenzione } \\
\text { del piede } \\
\text { diabetico dal } \\
\text { punto di vista } \\
\text { della persona } \\
\text { con DM. }\end{array}$ & $\begin{array}{l}\text { Progetto di } \\
\text { ampliament } \\
\text { o } \\
\text { universitari } \\
\text { o }\end{array}$ & $\begin{array}{l}\text { Azioni } \\
\text { specifiche, che } \\
\text { prevedono } \\
\text { propriamente } \\
\text { l'esame del } \\
\text { piede, il dialogo } \\
\text { comunicativo, } \\
\text { l'educazione } \\
\text { alla salute. }\end{array}$ \\
\hline $\begin{array}{l}\text { Alterações } \\
\text { nos pés do } \\
\text { idoso } \\
\text { hospitalizado: } \\
\text { um olhar } \\
\text { cuidadoso da } \\
\text { enfermagem } \\
2017\end{array}$ & $\begin{array}{l}\text { LILACS } \\
/ \\
\text { BDENF }\end{array}$ & $\begin{array}{l}\text { Esc. } \\
\text { Anna } \\
\text { Nery } \\
\text { Rev. } \\
\text { Enferm }\end{array}$ & $\begin{array}{l}\text { Caratterizzare } \\
\text { il profilo } \\
\text { podologico } \\
\text { degli anziani } \\
\text { ricoverati nei } \\
\text { reparti di un } \\
\text { ospedale } \\
\text { universitario; } \\
\text { identificare le } \\
\text { esigenze di } \\
\text { cura dei piedi } \\
\text { dei pazienti } \\
\text { anziani de } \\
\text { ospedalizzati; } \\
\text { e analizzare le } \\
\text { possibilità di } \\
\text { azioni degli } \\
\text { infermieri con } \\
\text { questi anziani. }\end{array}$ & Ospedale & $\begin{array}{l}\text { Rafforzare } \\
\text { legami con } \\
\text { questi pazienti } \\
\text { diabetici in } \\
\text { modo che } \\
\text { vengano al } \\
\text { posto per } \\
\text { eseguire } \\
\text { frequentemente } \\
\text { esami del piede } \\
\text { Formazione e } \\
\text { qualificazione } \\
\text { per aiutare } \\
\text { questi } \\
\text { problemi, } \\
\text { evitando } \\
\text { maggiori } \\
\text { complicazioni e } \\
\text { riducendo le }\end{array}$ \\
\hline
\end{tabular}




\begin{tabular}{|c|c|c|c|c|c|}
\hline & & & & & $\begin{array}{l}\text { future richieste } \\
\text { di assistenza }\end{array}$ \\
\hline $\begin{array}{l}\text { Atuação dos } \\
\text { enfermeiros } \\
\text { da estratégia } \\
\text { saúde da } \\
\text { família na } \\
\text { prevenção do } \\
\text { pé diabético } \\
2016\end{array}$ & $\begin{array}{l}\text { LILACS } \\
/ \\
\text { BDENF }\end{array}$ & $\begin{array}{l}\text { Rev. } \\
\text { Pesqui. } \\
\text { (Univ. } \\
\text { Fed. } \\
\text { Estado } \\
\text { Rio J., } \\
\text { Online) ; }\end{array}$ & $\begin{array}{l}\text { Identificare le } \\
\text { indicazioni } \\
\text { fornite dagli } \\
\text { infermieri alle } \\
\text { persone con } \\
\text { DM in cura dei } \\
\text { piedi; Indagare } \\
\text { la frequenza } \\
\text { dell'esame del } \\
\text { piede e gli } \\
\text { aspetti valutati; } \\
\text { verificare quali } \\
\text { attività } \\
\text { educazione } \\
\text { sanitaria } \\
\text { svolgono gli } \\
\text { infermieri per } \\
\text { le persone con } \\
\text { DM. }\end{array}$ & $\begin{array}{l}\text { Strategia } \\
\text { per la } \\
\text { salute della } \\
\text { famiglia }\end{array}$ & $\begin{array}{l}\text { Educazione } \\
\text { sanitaria per } \\
\text { diabetici, } \\
\text { esame dei piedi } \\
\text { di persone con } \\
\text { DM, } \\
\text { formazione di } \\
\text { gruppi } \\
\text { convivenza, } \\
\text { medicazioni in } \\
\text { tecniche } \\
\text { appropriate al } \\
\text { tipo di lesione } \\
\text { del piede. }\end{array}$ \\
\hline $\begin{array}{l}\text { A perspectiva } \\
\text { da } \\
\text { vulnerabilidad } \\
\text { e na } \\
\text { avaliação do } \\
\text { pé diabético } \\
\text { sob a ótica de } \\
\text { enfermeiros } \\
2016\end{array}$ & BDENF & $\begin{array}{l}\text { Cogitare } \\
\text { enferm }\end{array}$ & $\begin{array}{l}\text { Scrivi le } \\
\text { condizioni di } \\
\text { determinante } \\
\text { sociale in } \\
\text { salute, } \\
\text { identificate } \\
\text { dagli infermieri } \\
\text { come rilevanti } \\
\text { per l'istituzione } \\
\text { di vulnerabilità }\end{array}$ & $\begin{array}{ll}\text { Infermieri } & \\
\text { scelti a } \\
\text { caso a } \\
\text { Curitiba }\end{array}$ & $\begin{array}{lr}\text { Stimolare } & \text { il } \\
\text { diabetico ad } \\
\text { andare per la } \\
\text { valutazione } \\
\text { nutrizionale e } \\
\text { seguire } \\
\text { dieta. }\end{array}$ \\
\hline
\end{tabular}




$$
\begin{aligned}
& \text { della persona } \\
& \text { con diabete } \\
& \text { mellito. }
\end{aligned}
$$

Fonte: Autore.

Lo studio nei suoi obiettivi menziona tre tipi di cure eseguite dagli infermieri per i pazienti con piedi diabetici: il primo che è emerso più si basa su azioni volte all'educazione sanitaria come principio fondamentale della prevenzione della malattia e delle sue complicanze, la seconda cura si basa su tecniche di valutazione del piede nei diabetici come un modo per derivare da complicanze e la terza cura si concentra sugli infermieri come stimolante e motivante pazienti diabetici alla loro auto-cura con i piedi. Queste tre precauzioni saranno discusse nei loro temi in base agli studi selezionati.

\subsection{L'EDUCAZIONE SANITARIA COME FORMA DI PREVENZIONE}

Pertanto, dall'educazione sanitaria, il paziente può comprendere i motivi per cui il processo di guarigione delle ferite è lento, le ragioni delle principali complicanze e soprattutto le conoscenze relative alla prevenzione dei rischi. Gli infermieri dovrebbero essere in grado di riconoscere le esigenze del paziente e quindi contribuire a diagnosi infermieristiche accurate e sicure relative alla ferita nella sua fase.

Scain; Franzen; Hirakata (2018) riferisce che gli infermieri hanno mantenuto gli sforzi per eseguire l'educazione sanitaria con i pazienti, diventano persistenti e insistenti per continuare il processo educativo nei pazienti con piedi diabetici, utilizzare la creatività con lo sviluppo di tecnologie educative come cartelle, video, oltre ad essere disponibili a porre domande su alcune condizioni anormali con il paziente da tecnologie di social media come Whatsapp / telefono.

Camillo et al. (2016) menziona che il concetto di educazione sanitaria va oltre il portare dati o addirittura rimuovere i dubbi che sorgono, gli autori considerano 
l'educazione sanitaria un processo sistematico, continuo e permanente, volto alla formazione e allo sviluppo della consapevolezza critica del cittadino, attraverso la ricerca di soluzioni collettive ai problemi vissuti e la loro "reale partecipazione" all'esercizio del controllo sociale.

Vargas et al. (2017) porta l'educazione sanitaria come uno degli scopi principali delle attribuzioni degli infermieri alle cure, gli autori rafforzano che l'educazione sanitaria favorisce il paziente una postura più proattiva, dimostrando che il paziente dovrebbe avere sicurezza sulla cura del piede diabetico.

L'autonomia e la sicurezza delle cure sono state anche indicate come risultati dell'educazione sanitaria eseguita dagli infermieri nello studio di Pereira et al. (2017), gli autori riportano che l'educazione sanitaria diventa il principale meccanismo di prevenzione contro le malattie e le loro complicanze, in relazione al piede diabetico, l'educazione sanitaria promuove la sicurezza, la fiducia e l'autonomia del paziente.

La dimostrazione che l'educazione sanitaria è efficace è stata identificata in uno studio descrittivo di approccio quantitativo in cui ha dimostrato che l'educazione sanitaria ha portato ai pazienti una conoscenza teorica sulla malattia rispettando la cultura e il credo dei pazienti, e che la promozione dei processi educativi avviene non solo insieme, ma in modo individualizzato, togliendo i dubbi che permeano il paziente durante la consultazione infermieristica o anche in un dialogo informale tra il paziente e il professionista (DE OLIVEIRA et al., 2016).

Ma l'educazione sanitaria non è solo identificata come un processo di cura per i pazienti, gli infermieri dovrebbero anche essere attenti alla formazione e alle qualifiche, che possono anche essere considerate un'educazione sanitaria in modo più tecnico. Silva; Santo e Chibante (2017) affermano che gli infermieri dovrebbero sempre essere aggiornati a nuovi modi di prendersi cura dei piede diabetico, poiché i processi di trattamento sono sempre più studiati nella letteratura scientifica e portano innovazioni per la guarigione o addirittura la stadiazione delle ferite. 
Pertanto, l'educazione sanitaria, la formazione continua e la formazione continua sono forme di cura che gli infermieri utilizzano per migliorare il quadro clinico dei pazienti con piede diabetico.

\subsection{TECNICHE DI VALUTAZIONE DEL PIEDE DIABETICO}

Le conoscenze tecnico-scientifiche acquisite durante le fasi di formazione infermieristica e nell'ambito della formazione continua e permanente sono fattori rilevanti per il trattamento del piede diabetico, pertanto, spetta all'infermiere ricercare le conoscenze per eseguire tecniche di valutazione sicure per i pazienti con diabete come metodo di prevenzione e per i pazienti con piede diabetico come misure di prevenzione per le complicanze.

Arruda et al. (2019) afferma che l'esame fisico dei piedi diabetici sono procedure eseguite da infermieri al fine di evitare possibili complicazioni come: sviluppo di ulcere e amputazioni degli arti inferiori. Pertanto, l'ignoranza di questa tecnica induce i tassi più elevati di complicanze. Gli autori citati sottolineano in un altro momento che oltre alla tecnica non utilizzata a causa della mancanza di conoscenza dell'infermiere, le condizioni di lavoro influenzano anche la mancata esecuzione della tecnica di valutazione, queste condizioni sono indicate dagli autori come infrastrutture inadeguate e domanda di cure eccessive.

Vargas et al. (2017) porta nel suo studio un contesto simile allo studio precedentemente menzionato e aggiunge che gli infermieri hanno bisogno di maggiore formazione per quanto riguarda le tecniche di valutazione relative al piede diabetico, gli infermieri riferiscono anche che la direzione non offre materiali per eseguire queste tecniche e parlano di richieste eccessive.

Pereira et al. (2017) ritiene che dovrebbero essere intraprese azioni specifiche per rimodellare il sistema di assistenza per i pazienti con piede diabetico, tali azioni dovrebbero essere correlate oltre l'esame del piede, presentando una comunicazione efficace e un'educazione sanitaria. 
Leal et al. (2016) ritiene che gli infermieri possano avere ignoranza della materia a causa di diversi fattori e uno di questi è indicato dagli autori come l'elevata domanda di assistenza. Questa richiesta implica l'esaurimento fisico e mentale del professionista, che fornisce una mancanza di tempo per la qualificazione professionale. In un altro senso, gli autori menzionano che l'eccessiva domanda di un determinato problema fornisce al professionista di fare spazio all'educazione sanitaria permanente, in cui questo tipo di formazione propone trasformazioni nelle pratiche professionali basate su problemi esistenti nel processo del loro lavoro imparando a riflettere la pratica all'interno della loro realtà.

L'infermieristica porta con sé la funzione di trattare le malattie della pelle e le sue malattie adiacenti. Questo perché gli infermieri hanno nelle loro attribuzioni la cura del paziente e la necessità di espandere questa cura associata alle malattie legate alla pelle (DA SILVA BRANDAO et al., 2016; SA et al., 2016).

\subsection{IL MEDIATORE INFERMIERISTICO PER L'AUTOCURA DEI PAZIENTI CON PIEDE DIABETICO}

Parlare di cura di sé si riferisce a qualsiasi azione derivata dall'atto quotidiano dell'essere umano. Per quanto riguarda la cura di sé Arruda et al. (2019) riportano che i pazienti con piede diabetico tendono a generare un grande impatto economico, sociale, culturale, emotivo e biologico, che favorisce una maggiore dipendenza dagli altri per aiutare nelle attività della vita quotidiana e un impatto sulla loro cura di sé e sulla qualità della vita.

Scain; Franzen e Hirakata (2018) riferiscono che il paziente dovrebbe essere consapevole dello svolgimento delle proprie attività e che la cura di sé dovrebbe essere continua, uno dei modi per eseguire la cura di sé citati dagli autori erano: Igiene dei piedi; scelta di calzature appropriate; evitare di camminare a piedi nudi e gli altri.

A Vargas et al. (2017) porta come auto-cura il mantenimento del controllo glicemico, la partecipazione proattiva dei pazienti all'esecuzione di esami del piede, terapia, 
restrizione assoluta all'uso di tabacco e bevande, nutrizione, esercizio fisico, attività fisica e altre forme che contemplano lo studio precedentemente citato.

Un modo per ottenere che il paziente possa mirare alla cura di sé è citato dagli autori Silva; Santo e Chibante (2017) come rafforzamento dei legami tra paziente e operatore sanitario. Questo rafforzamento favorisce la sicurezza e la credibilità del paziente che eseguendo la cura di sé ai piedi in modo continuo e sistematico, minori sono le possibilità di rischio di complicanze.

L'autonomia e la sicurezza dimostrate dai pazienti con piede diabetico riducono al minimo i danni e i rischi di complicanze. Un paziente che è coinvolto nel trattamento della sua malattia tende ad espandere le sue conoscenze alla non dipendenza di altre persone, articolando nelle sue attività la cura di sé come forma di motivazione e dimostrazione di capacità di fronte alle avversità che coinvolgono il processo salutemalattia (DE OLIVEIRA et al., 2016).

A Fatima Bento et al. (2016), i pazienti con piede diabetico soffrono già di stigmi dalla loro condizione, il che rende difficile per loro avere cure adeguate, perché le condizioni che comportano la presa di distanza dall'unità sanitaria, le difficoltà nella mobilitazione, la scarsa conoscenza della malattia, consentono cure inadeguate ed evoluzione delle complicanze.

Tuttavia, Nascimento et al. (2016) menzionare che quando l'assistenza qualificata e umanizzata è fornita attraverso comportamenti efficaci, con un facile accesso ai servizi sanitari a cui tutte le azioni possono essere integrate a tutti i livelli di cura, la cura di sé senza rischio di complicanze.

\section{CONCLUSIONE}

Questo studio si è riflesso sulla cura di infermieri e professionisti nelle prestazioni di cura e cura per i pazienti con piede diabetico. Gli studi di cura rilevanti hanno identificato ciò che ha portato alla realizzazione di tre temi per la discussione dei risultati trovati. 
Lo scopo dello studio mirava a evidenziare l'assistenza fornita agli infermieri ai pazienti con piede diabetico, si sottolinea che è stata enfatizzata la cura degli infermieri e non di altre categorie di infermieristica. Gli studi hanno dimostrato che l'educazione sanitaria è la prima cura, il che non può essere contraddetto, poiché la letteratura sottolinea che l'educazione sanitaria è fondamentale per la prevenzione delle malattie.

Un altro tipo di cura si riflette nella conoscenza del professionista sul piede diabetico, di estremo bisogno e grande preoccupazione all'interno del sistema sanitario, poiché gli articoli hanno portato riflessioni sul fatto che gli infermieri dovrebbero essere più qualificati per indirizzare l'assistenza con più eccellenza al paziente.

Una cura importante sottolineata anche dagli studi e che è diretta direttamente con la formazione permanente o continua dei professionisti sono le tecniche di esame fisico, in questo tipo di cure, l'infermiere dovrebbe essere in grado di eseguire l'esame dei piedi diabetici con saggezza e sicurezza, oltre a incoraggiare anche i pazienti ad andare all'esame più frequentemente.

Per quanto riguarda le cure legate alla cura di sé del paziente, si è riflettuto sul fatto che l'igiene del piede, il controllo glicemico, il monitoraggio continuo con l'esame dei piedi, l'uso di calzature adeguate e altro intensificano una maggiore prevenzione contro le complicanze, compresa l'amputazione degli arti.

Si conclude, quindi, che lo studio ha identificato diverse forme di assistenza fornite dagli infermieri e che tutte queste forme sono importanti per la continuità del trattamento e la prevenzione e le complicanze, tuttavia, spetta all'infermiere essere un professionista che valorizza la qualità delle proprie cure sulla base di concetti teorici che portano a una formazione frequente.

\section{RIFERIMENTI}

AMERICAN DIABETES ASSOCIATION. Standards of medical care in diabetes. Diabetes Care, v. 40, n. suppl 1, p. s1-s128, 2017. 
ARRUDA, Cecilia et al. Tecnologia educativa para cuidados e prevenção do pé diabético/Educational technology for care and prevention of diabetic foot ulcers. Ciência, Cuidado e Saúde, v. 20, 2021.

ARRUDA, Luana Savana Nascimento de Sousa et al. Conhecimento do enfermeiro acerca dos cuidados com o pé diabético. Rev. enferm. UFPE on line, p. [1-8], 2019.

ASCHNER M, Pablo et al. Clinical practice guideline for the prevention, early detection, diagnosis, management and follow up of type 2 diabetes mellitus in adults. Colomb. Med., Cali, v. 47, n. 2, p. 109-130, junho. 2016.

CAMILLO, Bibiana Schultz et al. Ações de educação em saúde na atenção primária a gestantes e puérperas: revisão integrativa. Revista de enfermagem UFPE on line-ISSN: 1981-8963, v. 10, n. 6, p. 4894-4901, 2016.

CUBAS, Marcia Regina et al. Pé diabético: orientações e conhecimento sobre cuidados preventivos. Fisioterapia em movimento, v. 26, n. 3, 2017.

DE FÁTIMA BENTO, Leandra et al. A perspectiva da vulnerabilidade na avaliação do pé diabético sob a ótica de enfermeiros. Cogitare Enfermagem, v. 21, n. 1, 2016.

DE OLIVEIRA, Patrícia Simplício et al. Atuação dos enfermeiros da Estratégia Saúde da Família na prevenção do pé diabético Practice nurse family health strategy in the prevention of diabetic foot. Revista de Pesquisa Cuidado é Fundamental Online, v. 8, n. 3, p. 4841-4849, 2016.

DE SOUZA MENDONÇA, Sarah; MORAIS, Juliana de Sant'Anna; DE MOURA, Maria Catarina Gomes Gadelha. Proposta de um protocolo de avaliação fisioterapêutica para os pés de diabéticos. Fisioterapia em Movimento, v. 24, n. 2, 2017.

FELIX, Lidiany Galdino et al. Conhecimento de enfermeiros da atenção primária antes e após intervenção educativa sobre pé diabético. Revista Gaúcha de Enfermagem, v. 42, 2021. 
LEAL, Maria do Carmo et al. Atenção ao pré-natal e parto em mulheres usuárias do sistema público de saúde residentes na Amazônia Legal e no Nordeste, Brasil 2010. Rev. Bras. Saude Mater. Infant. Recife, v. 15, n. 1, p. 91-104, Mar. Available from \&lt; http://www.scielo.br/scielo.php?script=sci_arttext\&amp;pid=S151938292015000100091\&amp; Ing=en\&amp;nrm=iso\&gt;.

MENDES, Karina Dal Sasso; SILVEIRA, Renata Cristina de Campos Pereira; GALVÃO, Cristina Maria. Uso de gerenciador de referências bibliográficas na seleção dos estudos primários em revisão integrativa. Texto \& ContextoEnfermagem, v. 28, 2019.

MINAYO, Maria Cecília de Souza. Análise qualitativa: teoria, passos e fidedignidade. Ciência \& saúde coletiva, v. 17, p. 621-626, 2012.

MUZY, Jéssica et al. Prevalência de diabetes mellitus e suas complicações e caracterização das lacunas na atenção à saúde a partir da triangulação de pesquisas. Cadernos de Saúde Pública, v. 37, 2021.

NASCIMENTO, Vagner Ferreira do et al. Percepção de puérperas sobre as primeiras consultas de pré-natal no interior de Mato Grosso. Rev. enferm. UFPI, v.5, n. 1, p. 46-51, 2016.

ORGANIZAÇÃO MUNDIAL DE SAÚDE. Definition, diagnosis and classification of diabetes Mellitus and its complications. Geneva: OMS, 1999. Disponível em: https://apps.who.int/iris/bitstream/handle/10665/66040/WHO_NCD_NCS_99.2.pdf?s equence $=1$ \&isAllowed $=\mathrm{y}$

PEREIRA, Laiane de Fátima et al. Ações do enfermeiro na prevenção do pé diabético: o olhar da pessoa com diabetes mellitus. Rev. pesqui. cuid. fundam. (Online), p. 1008-1014, 2017.

SCAIN, Suzana Fiore; FRANZEN, Elenara; HIRAKATA, Vânia Naomi. Riscos associados à mortalidade em pacientes atendidos em um programa de prevenção do pé diabético. Revista Gaúcha de Enfermagem, v. 39, 2018. 
SILVA, Caline Oliveira da. Análise da qualidade de vida em indivíduos com Diabetes Mellitus Tipo 1. 2017. 34 f. Trabalho de conclusão de curso (Bacharelado em Farmácia) -Universidade de Brasília, Brasília, 2017. Disponível em: https://bdm.unb.br/handle/10483/19301

SILVA, Joziane Santos da; SANTO, Fátima Helena do Espírito; CHIBANTE, Carla Lube de Pinho. Alterações nos pés do idoso hospitalizado: um olhar cuidadoso da enfermagem. Escola Anna Nery, v. 21, 2017.

SOCIEDADE BRASILEIRA DE DIABETES. Tratamento e acompanhamento do diabetes Mellitus: Diretrizes da Sociedade Brasileira de Diabetes. 3.ed. Itapevi, SP: A. Araújo Silva Farmacêutica; 2017/2018. Disponível em: http://www.diabetes.org.br/ profissionais/images/2017/diretrizes/diretrizes-sbd-20172018.pdf.

VARGAS, Caroline Porcelis et al. Condutas dos enfermeiros da atenção primária no cuidado a pessoas com pé diabético. Rev. enferm. UFPE on line, p. 4535-4545, 2017.

Inviato: Ottobre, 2021.

Approvato: Dicembre 2021. 